\title{
Dislipidemia Secundária a Hipotiroidismo e Colestase
}

\section{Dyslipidemia Secondary to Hypothyroidism and Cholestasis}

Ana SAAVEDRA $\mathbb{1}^{1,2}$, Elisabete RODRIGUES ${ }^{1,2}$, Davide CARVALHO',2

Acta Med Port 2020 Mar;33(3):204-207 • https://doi.org/10.20344/amp.9944

RESUMO

Cerca de $28 \%$ dos doentes com dislipidemia apresentam na sua etiologia causas secundárias. A resolução de algumas destas causas pode levar à correção total da dislipidemia. Descreve-se o caso de uma doente do sexo feminino, 50 anos, com obesidade grau II e cirrose biliar primária referenciada por dislipidemia mista com mau controlo (sob estatina e fibrato) e com alterações analíticas hepáticas. No estudo efetuado constatou-se hipotiroidismo primário auto-imune. Após normalização da função tiroideia pelo tratamento com levotiroxina, além da suspensão da estatina e fibrato, verificou-se melhoria do perfil lipídico, embora mantendo hipercolesterolemia. Neste período de tempo foi-Ihe diagnosticada diabetes mellitus pelo que se instituiu de novo terapêutica com estatina (atorvastatina $10 \mathrm{mg}$ ), com normalização do perfil lipídico. Com este caso pretende-se salientar a importância da exclusão de causas secundárias de dislipidemia, nomeadamente o hipotiroidismo, bem como discutir aspectos particulares do tratamento com estatinas na doença hepática. Palavras-chave: Cirrose Hepática Biliar; Colestase; Dislipidemias; Hipotiroidismo

\section{ABSTRACT}

In about $28 \%$ of patients, dyslipidemia has a secondary cause. Many times, the treatment of these causes can lead to the total correction of dyslipidemia. We describe the case of a 50-year-old female patient with class II obesity and primary biliary cirrhosis, evaluated for mixed dyslipidemia with poor control (statins and fibrates were being administered) as well as abnormal liver tests. The investigation carried out revealed primary auto-immune hypothyroidism. After normalisation of thyroid function by treatment with levothyroxine, as well as suspending the administration of statins and fibrates, there was an improvement in the lipid profile, although hypercholesterolemia continued. During this time, the patient was diagnosed with diabetes and she re-commenced statin therapy (atorvastatin $10 \mathrm{mg}$ ), which resulted in a normal lipid profile being achieved. In this case, the authors set out to highlight the importance of excluding secondary causes of dyslipidemia - including hypothyroidism, and then go on to discuss particular aspects of statin therapy for liver disease. Keywords: Cholestasis; Dyslipidemias; Hypothyroidism; Liver Cirrhosis, Biliary

\section{INTRODUÇÃO}

Vários factores genéticos e ambientais podem interferir no normal metabolismo lipoproteico resultando em dislipidemia. Na maioria dos casos verifica-se uma componente familiar com hereditariedade poligénica e fortemente influenciada por factores ambientes. Menos frequentemente, ocorrem formas familiares monogénicas como a hipercolesterolemia familiar. ${ }^{1}$ Por outro lado, certas patologias e certos fármacos, podem causar secundariamente alterações lipídicas. ${ }^{1,2}$ Em $28 \%$ dos doentes com dislipidemia pode ser identificada uma causa secundária ${ }^{2,3}$ (Tabela 1), sendo fundamental o seu reconhecimento por várias razões. Em primeiro lugar, algumas causas secundárias representam importantes problemas de saúde e a dislipidemia pode ser uma das primeiras manifestações a permitir o seu diagnóstico. ${ }^{2}$ Em segundo, o tratamento da doença subjacente pode ser suficiente para corrigir a dislipidemia, reduzindo a necessidade de tratamento com fármacos hipolipemiantes. ${ }^{2,4} \mathrm{Em}$ terceiro, algumas situações de dislipidemia refratárias ao tratamento farmacológico podem dever-se a causas secundárias não devidamente identificadas e controladas. ${ }^{2}$ Apresentamos um caso que ressalta estes aspetos dada a contribuição de várias causas para a dislipidemia.

\section{CASO CLÍNICO}

Doente do sexo feminino, 50 anos, enviada à consulta de endocrinologia por dislipidemia mista (diagnóstico há 10 anos). Apresentava história de diabetes gestacional, obesidade grau II (IMC $\left.36,7 \mathrm{~kg} / \mathrm{m}^{2}\right)$, ex-fumadora desde há 18 anos (10 unidades maço/ano) e cirrose biliar primária (CBP) diagnosticada há três anos após avaliação de icterícia, associada a elevação de fosfatase alcalina (FA) e anticorpos antimitocondriais positivos (Apêndice 1: https:// www.actamedicaportuguesa.com/revista/index.php/amp/ article/view/9944/Apendice_01.pdf). Estava, por esse motivo, medicada com ácido ursodesoxicólico (AUDC). Negava história prévia de hipertensão arterial e de doença cardiovascular aterosclerótica bem como história familiar de dislipidemia ou doença cardiovascular. Sem prática de atividade física regular. Nos últimos três anos apresentara dislipidemia mista de difícil controlo apesar do tratamento com fármacos hipolipemiantes. Um ano antes da consulta desenvolveu miopatia, tendo suspendido temporariamente o tratamento com estatina (rosuvastatina). Na primeira consulta, encontrava-se medicada com rosuvastatina 10 $\mathrm{mg} / \mathrm{dia}$ e fenofibrato $145 \mathrm{mg} / \mathrm{dia}$, que foram suspensos dadas as alterações hepáticas [elevação das transaminases mais que três vezes o limite superior do normal (LSN)].

\footnotetext{
1. Serviço de Endocrinologia, Diabetes e Metabolismo. Centro Hospitalar de S. João. Porto. Portugal.

2. Instituto de Investigação e Inovação em Saúde. Faculdade de Medicina. Universidade do Porto. Porto. Portugal.

$\bowtie$ Autor correspondente: Ana Saavedra. amgsaavedra@gmail.com

Recebido: 14 de novembro de 2017 - Aceite: 14 de dezembro de 2018 | Copyright @ Ordem dos Médicos 2020
} 
Tabela 1 - Causas secundárias de dislipidemia

\begin{tabular}{l} 
Hipercolesterolemia \\
\hline - Hipotiroidismo \\
- Doença hepática colestática \\
- Síndrome nefrótico \\
- Tiazidas \\
\hline Hipertrigliceridemia \\
\hline - Diabetes mellitus \\
- Hipotiroidismo \\
- Dieta rica em hidratos de carbono \\
- Insuficiência renal \\
- Obesidade/insulinorresistência \\
- Lipodistrofias \\
- Estrogénios \\
- Etanol \\
- Bloqueadores beta-adrenérgicos \\
- Inibidores da protease \\
- Glucocorticóides \\
- Retinóides \\
- Antipsicóticos \\
- Riazidas \\
- Hiposinas sequestradoras de ácidos biliares \\
\hline
\end{tabular}

As análises realizadas para exclusão de causas secundárias permitiram diagnosticar hipotiroidismo primário auto-imune, embora a doente negasse qualquer sintomatologia sugestiva de hipotiroidismo com exceção de astenia ligeira. Foi iniciado tratamento com levotiroxina $100 \mu \mathrm{g} / \mathrm{dia}$, tendo sido posteriormente titulada a dose até normalização da função tiroideia (Apêndice 1: https://www.actamedicaportuguesa.com/revista/index.php/amp/article/view/9944/ Apendice_01.pdf).. Foi também detetada hiperglicemia intermédia (anomalia da glicemia em jejum e HbA1c 6,0\%). No seguimento, embora mantendo hipercolesterolemia, observou-se melhoria do perfil lipídico associado à normalização da função tiroideia. Por persistirem alterações da glicemia em jejum e $\mathrm{HbA} 1 \mathrm{c} 6,2 \%$, realizou prova de tolerância à glicose oral $(75 \mathrm{~g})$ para melhor caracterização do metabolismo dos hidratos de carbono, que permitiu o diagnóstico de diabetes mellitus (DM). Embora neste contexto clínico (obesidade grau II, dislipidemia, evolução insidiosa da glicemia em jejum) a hipótese mais provável fosse uma diabetes tipo 2, dado a presença de autoimunidade foram pedidos os anticorpos anti-GAD (negativos) permitindo excluir forma auto-imune de DM. Iniciou tratamento com meftormina 700 mg/dia, com bom controlo glicémico.

A par dos restantes factores de risco inicialmente apresentados pela doente (obesidade grau II; sedentarismo), o diagnóstico de DM tipo 2 (sem lesões de órgão alvo) veio agravar a categoria de risco cardiovascular, passando a apresentar um risco elevado. Considerando esta categoria de risco e a presença de transaminases em nível inferior a três vezes o LSN, iniciou atorvastatina $10 \mathrm{mg} / \mathrm{dia}$, tendo alcançado os objetivos terapêuticos.

\section{DISCUSSÃO}

As causas secundárias de dislipidemia (Tabela 1) são comuns e devem sempre ser excluídas. ${ }^{3,4}$ No caso apresentado coexistem várias patologias, que interferem de forma particular no metabolismo lipoproteico.

A cirrose biliar primária (CBP) é uma doença hepática crónica auto-imune caracterizada por perda progressiva dos ductos biliares intra-hepáticos e fibrose..$^{5} \mathrm{O}$ seu diagnóstico pode ser estabelecido pela presença de AMA positivos em doentes com elevação de FA, após exclusão de outras causas de colestase. ${ }^{6}$

Estando o fígado primariamente envolvido no metabolismo lipídico, é frequente a ocorrência de dislipidemia nas doenças colestáticas ( $1 / 3$ dos doentes), como a CBP. ${ }^{7}$ Tipicamente apresentam colesterol total muito elevado devido à presença de uma lipoproteína atípica $(L p X)$ e redução de recetores das C-LDL (rLDL) (Tabela 2). 5,8,9 Contudo, os níveis de c-LDL variam com a doença: no início, aumentam com a gravidade da CBP; em fases avançadas, pode haver redução por diminuição da síntese hepática de colesterol/ ácidos biliares e consequente diminuição da absorção intestinal de colesterol. ${ }^{7,8}$ Os níveis de c-HDL também podem estar mais elevados no início da doença e diminuídos em fases avançadas. Os triglicerídeos (TG) tipicamente estão aumentados. ${ }^{8} \mathrm{O}$ fenótipo lipídico na CBP é, assim, sugestivo de alto risco cardiovascular. Contudo, apesar de poucos estudos, o risco de doença coronária não parece aumentado. ${ }^{8,9}$ Segundo alguns autores, a LpX parece ter propriedades anti-aterogénicas. ${ }^{8,9}$

O AUDC está recomendado em todos os doentes com CBP, atrasando a progressão. ${ }^{5}$ Está associado a melhoria da colestase e excreção biliar de colesterol, redução da absorção intestinal de colesterol/ácidos biliares e consequente depleção intracelular hepática de colesterol, aumento da expressão/afinidade dos rLDL nos hepatócitos e redução da atividade da $H M G-C o A$ reductase., ${ }^{7,8}$ Muitas vezes o tratamento com AUDC é suficiente para corrigir a dislipidemia associada à colestase e as estatinas não são consideradas como primeira linha. ${ }^{5,9}$ No caso apresentado, as alterações lípidicas mantiveram-se apesar do tratamento com AUDC, e mesmo após início de fármacos hipolipemiantes, porque outra causa estava presente: hipotiroidismo. De facto, em $15 \%-20 \%$ dos casos de CBP coexiste doença tiroideia auto-imune..$^{5,8}$

O hipotiroidismo é uma outra causa secundária de dislipidemia, cuja prevalência em doentes com dislipidemia 
Tabela 2 - Alterações no metabolismo lipoproteico associadas à CBP

\section{1 - Presença de uma lipoproteína atípica: lipoproteína $X$}

- Resulta da regurgitação dos lípidos biliares para o plasma ou da acumulação sérica de fosfolípidos e colesterol livre por actividade reduzida de LCAT;

- Causa aumento directo do CT e indirecto por aumentar a síntese hepática de colesterol (diminui a captação hepática de colesterol e aumenta a actividade de HMG-CoA reductase*).

\section{2 - Redução dos receptores de LDL nos hepatócitos}

- Diminuição da depuração das partículas LDL pelos hepatócitos afectados pela CBP, aumentando os seus níveis séricos.

\section{3 - Redução da secreção de ácidos biliares devido à colestase}

- Resulta em diminuição da síntese hepática de ácidos biliares e de colesterol, mas este efeito é pouco significativo nos estádios iniciais/intermédios da CBP;

- Na CBP em fase avançada/terminal, estas alterações podem sobrepor-se à hipercolesterolemia da colestase. A redução marcada da síntese hepática de colesterol e da absorção intestinal do colesterol (por falta de ácidos biliares) podem resultar numa redução dos níveis de CT e LDL.

\section{4 - Diminuição da actividade da lípase hepática ${ }^{\dagger}$}

- Aumento dos triglicerídeos e lipoproteínas ricas em triglicerídeos.

\section{5 - Diminuição da actividade da LCAT}

- Apesar da redução da actividade da lípase hepática, verifica-se redução concomitante da LCAT, o que contribui para uma redução dos níveis de HDL nas fases mais avançadas da CBP

CBP: cirrose biliar primária; CT: colesterol total; HMGCoA reductase: 3-hidroxi-3-methyl-glutaril-CoA redutase; HDL: high density lipoprotein; LCAT: lecithin-cholesterol acyltransferase; LDL: low density lipoprotein.

*Enzima passo-limitante da via de síntese hepática de colesterol. † Enzima localizada na membrana dos hepatócitos e responsável pela hidrólise de triglicerídeos e fosfolípidos presentes nos remanescentes de quilomicrons, IDL (convertendo-os em LDL) e HDL (convertendo as grandes partículas HDL2 (ricas em triglicerídeos e ésteres de colesterol) em $\mathrm{HDL}_{3}$ ). ‡ Enzima produzida no fígado e presente na membrana das HDL. Está envolvida no transporte reverso do colesterol catalisando a síntese de ésteres de colesterol, o que permite a transferência de colesterol livre da membrana das HDL para o interior hidrofóbico da lipoproteína. Deste modo, em condições normais diminui a concentração de colesterol livre na membrana das HDL, facilitando o uptake contínuo de colesterol livre.

Tabela 3 - Alterações no metabolismo lipoproteico associadas ao hipotiroidismo

\section{1 - Diminuição do receptor das LDL}

- Resulta da diminuição do efeito directo da T3 sobre o promotor do gene do rLDL (via ligação a TREs) e por diminuição do controlo da T3 sobre SREBP2* (mecanismo indirecto).

- Ocorre uma diminuição da clearance das LDL e IDL, havendo aumento do número de partículas em circulação, as quais podem sofrer oxidação, aumentando a quantidade de partículas LDL oxidadas e promovendo a formação de células espumosas.

\section{2 - Diminuição da transcrição/actividade da HMG-CoA reductase}

- Ocorre por diminuição do controlo da T3 via SREBP2.

- Resulta em diminuição da síntese de colesterol (efeito menos marcado relativamente às outras alterações induzidas pelo hipotiroidismo).

\section{3 - Diminuição da excreção de colesterol pela redução da síntese de ácidos biliares}

- Ocorre por diminuição da enzima colesterol 7-alfa-hidroxilase†.

\section{4 - Diminuição da actividade da lipoproteína lípase}

- Efeito mediado por aumento de ANGPTL3 (inibidor potente da LPL) e diminuição da APO A5.

- Resulta na diminuição da clearance das lipoproteínas ricas em TG e consequentemente aumento dos níveis de TG, VLDL e por vezes quilomicronemia.

\section{5 - Diminuição da actividade da lípase hepática}

- Resulta em níveis elevados de HDL (particularmente das partículas HDL2).

\section{6 - Diminuição da actividade da CETP ${ }^{\ddagger}$}

- Contribui para os níveis elevados de HDL.

\section{7 - Diminuição da actividade da LCAT}

- Diminui a conversão de colesterol livre em ésteres de colesterol, reduzindo a captação de colesterol livre pelas partículas HDL.

ANGOPTL3: angioprotein-like 3; APO A5: apoproteina 5, CETP: cholesteryl-esters transfer protein; HDL: high density lipoprotein; HMGCoA reductase: 3-hidroxi-3-methyl-glutaril-CoA redutase; IDL: intermediate density lipoprotein; LCAT: lecithin-cholesterolacyltransferase; LDL: low density lipoprotein; LPL: lipase lipoprotein; rLDL: receptor das LDL; SREBP2: sterol regulatory element binding protein 2; TG: triglicerídeos; TREs: thyroid hormone responsive elements; T3: triiodotironina; VLDL: very low density lipoprotein.

* Sensor localizado no reticulo endoplasmático, que é activado quando os níveis intracelulares de colesterol estão diminuídos. Após activação migra para o núcleo onde actua com factor de transcrição, levando à transcrição do gene do rLDL e outros genes envolvidos na síntese de colesterol. A sua expressão é regulada pelas hormonas tiróideias, estando diminuída no hipotiroidismo. † Enzima passo-limitante na síntese de ácidos biliares a partir de colesterol. A transcrição do seu gene é regulada pela ligação da T3 a TRE. ‡ Enzima que medeia a transferência de ésteres de colesterol das HDL para as VLDL, quilomicrons e LDL.

é de $2,8 \%{ }^{10}$ As hormonas tiroideias têm múltiplos efeitos no metabolismo lipídico. A hipercolesterolemia associada ao hipotiroidismo deve-se a redução dos rLDL e ausência da ação das hormonas tiroideias sobre os ácidos biliares (Tabela 3). ${ }^{11,12}$ Estas alterações revertem após tratamento com levotiroxina, exceto se o doente tiver dislipidemia 
subjacente. Assim, não devem ser iniciadas estatinas sem antes ser reestabelecido eutirodismo. ${ }^{12,13}$ Por outro lado, tendo em conta o possível risco de miopatia/rabdomiólise associado às estatinas e que o hipotiroidismo também se associa a aumento de creatina cinase, a sua coexistência pode potenciar este risco. ${ }^{12} \mathrm{Na}$ doente, verificou-se melhoria da dislipidemia após normalização da função tiroideia. Este aspeto reforça a importância da exclusão das causas secundárias, nomeadamente o hipotiroidismo, na avaliação do doente com dislipidemia. Nesse sentido, a avaliação da função tiroideia deverá ser sempre incluída no estudo analítico inicial destes doentes. ${ }^{4}$

Após diagnóstico de DM, a doente passou a apresentar risco cardiovascular elevado, requerendo tratamento com estatinas para alcançar o objectivo terapêutico. A CBP poderia condicionar o início de estatinas, mas nessa altura a doente apresentava elevação de transaminases inferior a três vezes o LSN, não contra-indicando a sua utilização. Um outro aspeto a salientar diz respeito à possível influência das hormonas tiroideias nos níveis séricos das transaminases. Ligeiras elevações dos níveis de AST e ALT podem ser encontrados nos doentes com hipotiroidismo, sendo geralmente reversíveis após a introdução do tratamento com levotiroxina. ${ }^{14}$ Isto poderá explicar a diminuição nos níveis de transaminases que se observou nesta doente, passando de níveis significativamente elevados para valores compatíveis com a introdução de estatina.

No entanto, existe pouca evidência quanto ao uso de estatinas na doença hepática crónica. ${ }^{8} \mathrm{Na}$ doença avançada, o estado de equilíbrio e a concentração máxima das estatinas podem ser mais elevados, mas não parece haver diferenças na doença ligeira. ${ }^{15}$ Nas situações colestásticas, pode haver acumulação das estatinas que sofrem excreção biliar (lovastatina, sinvastatina, atorvastatina, rosuvastatina), pelo que devem ser evitadas ou usadas criteriosamente. ${ }^{8,15}$ No entanto, estudos mais recentes têm demonstrado que a utilização de estatinas, nomeadamente sinvastatina e atorvastatina, parece ser segura em doentes com CBP. ${ }^{15}$

\section{REFERÊNCIAS}

1. Durrington P. Dyslipidaemia. Lancet. 2003;362:717-31.

2. Vodnala D, Rubenfire M, Brook RD. Secondary causes of dyslipidemia. Am J Cardiol. 2012;110:823-5.

3. Melmed S, Polonsky K, Larsen PR, Kronenberg H. Williams textbook of endocrinology. $13^{\text {th }}$ ed. Elsevier. 2015. Section VIII - Chapter 37.

4. Jellinger PS, Handelsman Y, Rosenblit PD, Bloomgarden ZT, Fonseca VA, Garber AJ, et al. American Association of Clinical Endocrinologists and American College of Endocrinology Guidelines for Management of Dyslipidemia and Prevention of Cardiovascular Disease. Endocr Pract. 2017;23:S1-87.

5. Silveira MG, Lindor KD. 38-year-old woman with abnormal liver enzymes and hyperlipidemia. Mayo Clin Proc. 2009;84:551-4.

6. Carey EJ, Ali AH, Lindor KD. Primary biliary cirrhosis. Lancet. 2015;386:1565-75

7. Balmer ML, Dufour JF. Treatment of hypercholesterolemia in patients with primary biliary cirrhosis might be more beneficial than indicated. Swiss Med Wkly. 2008;138:415-9.

8. Sorokin A, Brown JL, Thompson PD. Primary biliary cirrhosis,
Relativamente à doente do caso clínico e atendendo ao risco cardiovascular que apresentava, optou-se pela introdução de uma estatina de potência moderada, tendo também em consideração que o perfil hepático não contra-indicava a sua utilização.

\section{CONCLUSÃO}

Em conclusão, várias patologias podem causar dislipidemia ou contribuir para a falência terapêutica, sendo frequentemente esquecidas. Importa salientar que: (i) muitas causas secundárias não requerem tratamento com estatinas pois as alterações podem ser reversíveis; (ii) o hipotiroidismo aumenta o risco de rabdomiólise, devendo ser sempre excluído; (iii) a dislipidemia associada à CBP não parece associar-se a risco aterosclerótico aumentado; (iv) e por fim, é possível o uso de estatinas na doença hepática ajustando à fase da doença, embora seja necessária mais evidência.

\section{PROTECÇÃO DE PESSOAS E ANIMAIS}

Os autores declaram que os procedimentos seguidos estavam de acordo com os regulamentos estabelecidos

\section{CONFIDENCIALIDADE DOS DADOS}

Os autores declaram ter seguido os protocolos do seu centro de trabalho acerca da publicação de dados.

\section{CONSENTIMENTO DO DOENTE}

Obtido.

\section{CONFLITOS DE INTERESSE}

Os autores declaram a inexistência de conflitos de interesse na realização do presente trabalho.

\section{FONTES DE FINANCIAMENTO}

Este trabalho foi realizado sem contributo de subsídios ou bolsas.

hyperlipidemia, and atherosclerotic risk: a systematic review. Atherosclerosis. 2007;194:293-9.

9. Foley KF, Silveira MG, Hornseth JM, Lindor KD, McConnell JP. A patient with primary biliary cirrhosis and elevated LDL cholesterol. Clin Chem. 2009;55:187-91.

10. Tsimihodimos V, Bairaktari E, Tzallas C, Miltiadus G, Liberopoulos E, Elisaf $M$. The incidence of thyroid function abnormalities in patients attending an outpatient lipid clinic. Thyroid. 1999;9:365-8.

11. Duntas LH, Brenta G. The effect of thyroid disorders on lipid levels and metabolism. Med Clin North Am. 2012;96:269-81.

12. Rizos CV, Elisaf MS, Liberopoulos EN. Effects of thyroid dysfunction on lipid profile. Open Cardiovasc Med J. 2011;5:76-84.

13. Jin $T$, Teng $X$. Update on lipid metabolismo and thyroid disorders. J Endocrinol Diabetes Obes. 2014;2:1043-8.

14. Saha B, Maity C. Alteration of serum enzymes in primary hypothyroidism. Clin Chem Lab Med. 2002;40:609-11.

15. Herrick C, Litvin M, Goldberg AC. Lipid lowering in liver and chronic kidney disease. Best Pract Res Clin Endocrinol Metab. 2014;28:339-52. 